higher prevalence of syndesmophytes ( $44 \%$ vs $36 \%, \mathrm{p}=0.06)$ and hip involvement $(20 \%$ vs $15 \%, p=0.09)$ was observed in the group of $A U$.

Regarding CV risk features, no differences were observed in the prevalence of CV risk factors and events (Table 1). Patients with AU showed a higher cIMT in the crude analysis ( $665 \pm 156 \mathrm{~mm}$ vs $640 \pm 142 \mathrm{~mm}, \mathrm{p}=0.047)$, but no significant differences were observed after adjustment by age and sex $(\mathrm{p}=0.6)$. Prevalence of carotid plaques was comparable in both groups (32\% Vs $32 \%, p=0.84)$.

Table 1.

\begin{tabular}{|c|c|c|c|c|}
\hline \multirow[b]{2}{*}{ Men/Women, $\mathrm{n}$} & \multicolumn{2}{|c|}{$\begin{array}{l}\text { axSpA without axSpA with } \\
\text { uveitis }(n=709) \text { uveitis }(n=177)\end{array}$} & p & \multirow[t]{2}{*}{$\begin{array}{l}\mathrm{P} \text { (adjusted } \\
\text { model) }\end{array}$} \\
\hline & $477 / 232$ & $122 / 55$ & 0.68 & \\
\hline $\begin{array}{l}\text { Mean age (years) } \pm S D \text { at the time of } \\
\text { study }\end{array}$ & $48 \pm 13$ & $50 \pm 11$ & 0.049 & \\
\hline AS/nr-AxSpa & $541 / 168$ & $160 / 17$ & 0.000 & \\
\hline \multicolumn{5}{|l|}{ History of $\mathrm{CV}$ risk factors, $\mathrm{n}(\%)$} \\
\hline $\begin{array}{l}\text { Current smoker } \\
\text { Obesitty }\end{array}$ & $247(35)$ & $50(28)$ & 0.096 & \\
\hline Dyslipemia & $233(33)$ & $63(36)$ & 0.48 & \\
\hline Hypertension & $188(27)$ & $50(28)$ & 0.63 & \\
\hline Diabetes Mellitus & $50(7)$ & $14(8)$ & 0.69 & \\
\hline Chronic Kidney Disease & $18(3)$ & $4(2)$ & 0.99 & \\
\hline History of cardiovascular events, $\mathrm{n}(\%$ & $29(4)$ & $12(7)$ & 0.13 & \\
\hline \multicolumn{5}{|c|}{ Structural damage at the time of study } \\
\hline Presence of syndesmophytes, n (\%) & $253(36)$ & $77(44)$ & 0.063 & \\
\hline mSASSS & $5(1-15)$ & $6(0-16)$ & 0.31 & \\
\hline Severe sacroiliitis (grade 3,4$), \mathrm{n}(\%)$ & $348(49)$ & $122(69)$ & 0.000 & $0.000^{*}$ \\
\hline \multicolumn{5}{|l|}{ Carotid US data at the time of study } \\
\hline Carotid plaques, n (\%) & 225 (32) & $57(32)$ & 0.84 & \\
\hline IMT (mm) & $640 \pm 142$ & $665 \pm 156$ & 0.047 & $70.6^{\star *}$ \\
\hline IMT > $=0.900 \mathrm{~mm}$ & $36(5)$ & $10(6)$ & 0.72 & \\
\hline
\end{tabular}

*: adjusted by age, disease duration and AS/nr-axSpA ratio

**: adjusted by age and sex

Abbreviations: $\mathrm{AS}=$ ankylosing spondylitis. $\mathrm{AxSpA}=$ axial spondylitis. $\mathrm{CV}=$ cardiovascular. IMT

$=$ intima-media wall thickness. $\mathrm{Nr}$-axSpA $=$ no-radiographic axial spondylitis

Conclusion: The presence of $\mathrm{AU}$ does not confer additional $\mathrm{CV}$ risk to axSpA patients, although it is associated with a more severe structural damage in our series. REFERENCES:

[1] Conkar S, Güven Yılmaz S, Koska İÖ, Berdeli A, Mir S. Evaluation of development of subclinical atherosclerosis in children with uveitis. Clin Rheumatol. 2018 May;37(5):1305-1308.

Disclosure of Interests: None declared

DOI: 10.1136/annrheumdis-2021-eular.909

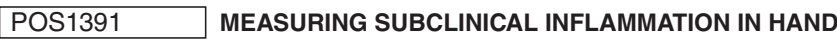 AND FOREFOOT IN PATIENTS WITH ARTHRALGIA USING 1.5T OR 3.0T MRI: DOES FIELD STRENGTH MATTER?}

D. Krijbolder ${ }^{1}$, M. Verstappen ${ }^{1}$, F. Wouters ${ }^{1}$, L. R. Lard ${ }^{2}$, P. D. De Buck ${ }^{3}$, J. Verisvan Dieren ${ }^{4}$, M. Reijnierse ${ }^{5}, \mathrm{H}$. Bloem ${ }^{5}, \mathrm{~A}$. Van der Helm - van Mil ${ }^{1,6}$. ${ }^{1}$ Leiden University Medical Center, Department of Rheumatology, Leiden, Netherlands; ${ }^{2}$ LangeLand Ziekenhuis, Department of Rheumatology, Zoetermeer, Netherlands; ${ }^{3}$ Haaglanden Medical Center, Department of Rheumatology, Den Haag, Netherlands; ${ }^{4}$ Reumazorg Zuid West Nederland, Department of Rheumatology, Goes, Netherlands; ${ }^{5}$ Leiden University Medical Center, Department of Radiology, Leiden, Netherlands; ${ }^{6}$ Erasmus Medical Center, Department of Rheumatology, Rotterdam, Netherlands

Background: Magnetic resonance imaging (MRI) of small joints sensitively detects inflammation. MRI-detected subclinical inflammation, and tenosynovitis in particular, has been shown predictive for RA development in patients with arthralgia. These scientific data are mostly acquired on 1.0T-1.5T MRI scanners. However, 3.0T $\mathrm{MRI}$ is nowadays increasingly used in practice. Evidence on the comparability of these field strengths is scarce and it has never been studied in arthralgia where subclinical inflammation is subtle. Moreover, comparisons never included tenosynovitis, which is, of all imaging features, the strongest predictor for progression to RA. Objectives: To determine if there is a difference between 1.5T and 3.0T MRI in detecting subclinical inflammation in arthralgia patients.

Methods: 2968 locations (joints, bones or tendon sheaths) in hands and forefeet of 28 arthralgia patients were imaged on both 1.5T and 3.0T MRI. Two independent readers scored for erosions, osteitis, synovitis (according to RAMRIS) and tenosynovitis (as described by Haavaardsholm et al.). Scores were also summed as total inflammation (osteitis, synovitis and tenosynovitis) and total RAMRIS (erosions, osteitis, synovitis and tenosynovitis) scores. Interreader reliability (comparing both readers) and field strength agreement (comparing 1.5T and 3.0T) was assessed with interclass correlation coefficients (ICCs). Next, field strength agreement was assessed after dichotomization into presence or absence of inflammation. Analyses were performed on patient- and location-level.
Results: ICCs between readers were excellent (>0.90). Comparing 1.5 and 3.0T revealed excellent ICCs of 0.90 (95\% confidence interval 0.78-0.95) for the total inflammation score and $0.90(0.78-0.95)$ for the total RAMRIS score. ICCs for individual inflammation features were: tenosynovitis: $0.87(0.74-0.94)$, synovitis $0.65(0.24-0.84)$ and osteitis 0.96 (0.91-0.98). The field strength agreement on dichotomized scores was $83 \%$ for the total inflammation score and $89 \%$ for the total RAMRIS score. Of the individual features, agreement for tenosynovitis was the highest (89\%). Analyses on location- level showed similar results.

Conclusion: Agreement of subclinical inflammation scores on 1.5T and 3.0T were good to excellent, in particular for tenosynovitis. This suggests that scientific evidence on predictive power of MRI in arthralgia patients, obtained on 1.5T, can be generalized to 3.0T when this field strength would be used for diagnostic purposes in daily practice. Disclosure of Interests: None declared DOI: 10.1136/annrheumdis-2021-eular.1417

\section{POS1392 \\ ECHOCARDIOGRAPHIC FINDINGS IN PSORIATIC ARTHRITIS, RHEUMATOID ARTHRITIS AND CONTROLS}

A. B. Rodriguez-Romero ${ }^{1}$, J. R. Azpiri-López ${ }^{2}$, I. J. Colunga-Pedraza ${ }^{1}$, D. Á. Galarza-Delgado ${ }^{1}$, N. Guajardo-Jauregui ${ }^{1}$, S. Lugo-Perez ${ }^{2}$, A. Cárdenas ${ }^{1}$, H. Azpiri-Diaz ${ }^{2}$, O. A. Cepeda-Ayala ${ }^{2} .{ }^{1}$ Hospital Universitario "Dr. José Eleuterio González", Universidad Autónoma de Nuevo León, Rheumatology Service, Monterrey, Mexico; ${ }^{2}$ Hospital Universitario "Dr. José Eleuterio González" Universidad Autónoma de Nuevo León, Cardiology Service, Monterrey, Mexico

Background: Psoriatic arthritis (PsA) is a chronic inflammatory arthropathy associated with cardiovascular abnormalities (1). The echocardiography is a non-invasive tool useful in the detection of cardiac abnormalities, which may be the only manifestation of cardiac involvement preceding a global dysfunction. However, echocardiographic differences between PsA patients, rheumatoid arthritis (RA) patients, and controls have not yet been well described.

Objectives: To analyze the echocardiographic parameters in PsA patients and to compare them with RA patients and controls.

Methods: This cross-sectional, observational and comparative study, included thirty-six patients (nineteen in each group), aged 40-75 years, with PsA and RA who fulfilled the CASPAR (Classification criteria for Psoriatic Arthritis) and ACR/EULAR 2010 classification criteria, respectively, matched by age, gender and comorbidities with nineteen healthy controls. Exclusion criteria were a poor echocardiographic window, patients with a previous atherosclerotic cardiovascular disease (ischemic heart disease, cerebrovascular accident or peripheral arterial disease), and pregnancy. Transthoracic echocardiogram was performed and reviewed by 2 board-certified cardiologists, in all study subjects. Comparisons were done with X2, Kruskall Wallis or ANOVA.

Results: There were not differences in the demographic characteristics between groups (Table 1). When comparing echocardiographic findings a statistically significant difference was found in the prevalence of diastolic dysfunction, being more prevalent in PsA and RA patients compared with controls (52.6\% vs $52.6 \%$ vs $5.3 \%$, $\mathrm{p}=0.002)$, likewise the presence of mild mitral valve regurgitation was higher $(84.2 \%$ vs $53.6 \%$ vs $10.5 \%, p=0.001)$ and mild pulmonary valve regurgitation $(68.4 \%$ vs $10.5 \%$ vs $0 \%, p=0.001$ ). Prevalence of abnormal left ventricular geometry was higher in PsA and RA patients than controls ( $68.4 \%$ vs $63.2 \%$ vs $21.1 \%, p=0.006)$

Table 1. Comparison of demographic characteristics and echocardiographic findings between patients with PsA, RA and controls.

\begin{tabular}{|c|c|c|c|c|}
\hline & $\begin{array}{l}\text { PsA } \\
(n=19)\end{array}$ & $\begin{array}{l}\text { RA } \\
(n=19)\end{array}$ & $\begin{array}{l}\text { Controls } \\
(n=19)\end{array}$ & $p$ \\
\hline Age, years $\pm S D$ & $54.7 \pm 7.7$ & $55.4 \pm 9.9$ & $55.3 \pm 5.9$ & NS \\
\hline Female, n (\%) & $11(57.9)$ & $11(57.9)$ & $11(57.9)$ & NS \\
\hline Diabetes Mellitus & $4(21.1)$ & $3(15.8)$ & $2(10.5)$ & NS \\
\hline Hypertension & $10(52.6)$ & $8(42.1)$ & $7(36.8)$ & NS \\
\hline Dyslipidemia & $10(52.6)$ & $4(21.1)$ & $7(36.8)$ & NS \\
\hline Active smoking & $4(21.1)$ & $2(10.5)$ & $5(26.3)$ & NS \\
\hline Disease duration, years (p25-p75) & $6(4-14)$ & $7(5-18)$ & - & NS \\
\hline $\begin{array}{l}\text { DAS28-CRP, mean } \pm \text { SD } \\
\text { Echocardiographic findigs }\end{array}$ & $2.2 \pm 0.8$ & $3.1 \pm .8$ & - & 0.003 \\
\hline Diastolic dysfunction, n (\%) & $10(52.6)$ & $10(52.6)$ & $1(5.3)$ & 0.002 \\
\hline LV mass index, g/m² (p25-p75) & $78.9(55.9-86.9)$ & $73.7(61.0-85.7)$ & $69.5(52.0-98.7)$ & NS \\
\hline $\mathrm{LVEF} \pm$ mean SD & $62.3 \pm 6.1$ & $59.7 \pm 8.6$ & $62.9 \pm 6.1$ & NS \\
\hline TAPSE, $\mathrm{cm} \pm \mathrm{SD}$ & $21.8 \pm 2.7$ & $22.4 \pm 2.7$ & $23.7 \pm 3.1$ & NS \\
\hline Mild aortic regurgitation, $\mathrm{n}(\%)$ & $5(26.3)$ & $4(21.1)$ & $1(5.3)$ & NS \\
\hline Mild mitral regurgitation, $\mathrm{n}(\%)$ & $16(84.2)$ & $10(52.6)$ & $2(10.5)$ & $<0.001$ \\
\hline Mild pulmonary regurgitation, $\mathrm{n}(\%)$ & ) $13(68.4)$ & $2(10.5)$ & $0(0)$ & $<0.001$ \\
\hline Mild tricuspid regurgitation, $\mathrm{n}(\%)$ & $15(83.3)$ & $13(76.5)$ & $11(57.9)$ & NS \\
\hline LV geometry alterations, $\mathrm{n}(\%)$ & $13(68.4)$ & $12(63.2)$ & $4(21.1)$ & 0.006 \\
\hline Concentric remodeling, $n(\%)$ & $12(63.2)$ & $10(52.6)$ & $4(21.1)$ & 0.025 \\
\hline
\end{tabular}

NS, non-significant; DAS28-CRP, disease activity score using 28 joints and $C$ reactive protein; $L V$, left ventricular; LVEF, left ventricular ejection fraction; TAPSE, tricuspid annular plane systolic excursion 
Conclusion: This study shows the high prevalence of echocardiographic alterations in PsA patients compared to the general population, of the same magnitude as patients with RA. We emphasize the value of an echocardiogram for a complete cardiovascular evaluation and early detection of cardiac abnormalities in these patients.

REFERENCES:

[1] Shang Q, Tam LS, Yip GW, et al. High prevalence of subclinical left ventricular dysfunction in patients with psoriatic arthritis. J Rheumatol 2011;38(7):136370. doi: 10.3899/jrheum.101136

Disclosure of Interests: None declared

DOI: 10.1136/annrheumdis-2021-eular.1439

\section{POS1393 QUANTITATIVE AUTOFLUORESCENCE FINDINGS IN PATIENTS UNDERGOING HYDROXYCHLOROQUINE TREATMENT}

S. Parrulli ${ }^{1}$, M. Cozzi ${ }^{1}$, M. Airaldi ${ }^{1}$, F. Romano ${ }^{1}$, F. Viola ${ }^{2}$, P. Sarzi-Puttini ${ }^{3}$, G. Staurenghi ${ }^{1}$, A. Invernizzi ${ }^{1,4} .{ }^{1}$ Sacco Hospital, University of Milan, Eye Clinic, Department of Biomedical and Clinical Science "Luigi Sacco", Milan, Italy; ${ }^{2}$ University of Milan, IRCCS-Ca Granda Fondazione Ospedale Maggiore Policlinico, Ophthalmological Unit, Department of Clinical Sciences and Community Health, Milan, Italy; ${ }^{3}$ ASST Fatebenefratelli L. Sacco University Hospital, Rheumatology Unit, Milan, Italy; ${ }^{4}$ Sydney Medical School, The University of Sydney, Save Sight Institute, Discipline of Ophthalmology, Sydney, Australia

Background: Hydroxychloroquine (HCQ) is a relatively safe and effective drug widely used as primary or adjunctive treatment for several rheumatological and dermatological disorders ${ }^{1}$. HCQ modulates immune response through several mechanisms and has a tropism for pigmented ocular tissues, particularly retinal pigment epithelium (RPE) ${ }^{2}$. Its accumulation within RPE cells can lead to sight threatening retinal toxicity, with bull's eye maculopathy (BEM) representing its advanced phenotype. ${ }^{3}$ Quantitative Auto-Fluorescence (qAF) is an imaging modality that allows the measurement of retinal auto-fluorescence following short-wavelength light (488nm) excitation of retinal fluorophores (lipofuscin). ${ }^{4}$ Two recent studies have focused on qAF values in patients treated with $\mathrm{HCQ}^{5,6}$. In both cases qAF was increased in eyes with BEM. Furthermore, Reichel et al. ${ }^{6}$ were able to detect increased values of qAF in patients without BEM as early as 6 months after the start of $\mathrm{HCQ}$ treatment using an experimental imaging analysis procedure.

Objectives: To measure quantitative autofluorescence (qAF) in patients under treatment with hydroxychloroquine (HCQ) with no apparent signs of retinal toxicity and to compare it with that of untreated subjects.

Methods: Consecutive patients at risk for the development of $\mathrm{HCQ}$ retinal toxicity (duration of treatment $>5$ years or daily $\mathrm{HCQ}$ dose $>5 \mathrm{mg} / \mathrm{kg}$ of actual body weight (ABW) and/or renal insufficiency) ${ }^{7}$ but no alterations on Spectral Domain - Optical Coherence Tomography, Short-Wavelength Autofluorescence and 10-2 Visual Field examination were recruited. Healthy subject matched by age and sex were also enrolled in the study. All subjects underwent qAF measurements in one eye. Images were analyzed using the conventional qAF grid by Delori calculating the qAF of 8 sectors of the intermediate ring and the mean of those values ( $\mathrm{qAF}_{8}$ ). Results: Thirty-nine patients treated with HCQ (38 females, mean age 52,1 \pm 8,6 years) and 39 untreated subjects (38 females, mean age 51,2 $\pm 8,6$ years). In both $\mathrm{HCQ}$ patients and untreated subjects, $\mathrm{qAF}_{8}$ was positively correlated with age $(p=0.004)$ (Figure 1). Although $\mathrm{HCQ}$ patients showed a higher mean $\mathrm{qAF}_{8}$ compared to untreated subjects $(294,7 \pm 65,3$ vs $268,9 \pm 57,5)$, the difference was not significant $(p=0.068)$. HCQ patients showed significantly higher mean qAF values in the inferior-temporal, inferior and inferior-nasal sectors of the intermediate ring of qAF grid compared to untreated subjects (all $p<0.05$ ).

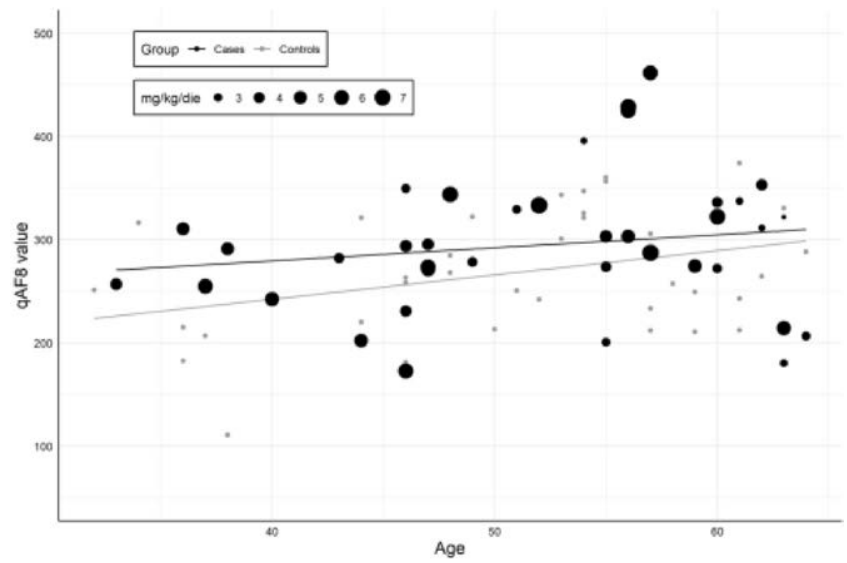

Figure 1. Visual representation of a model predicting the standardized qAF values as influenced by age and HCQ daily dose/ABW, calculated for a treatment duration of 15 years.
Conclusion: These results suggest a possible preclinical increase of qAF values in inferior parafoveal sectors probably induced by HCQ exposure. Further studies are required to improve our understanding of preclinical stages of $\mathrm{HCQ}$ retinopathy and the possible role of $\mathrm{qAF}$ in the HCQ toxicity screening.

\section{REFERENCES:}

[1] Haładyj, E., Sikora, M., Felis-Giemza, A. \& Olesińska, M. Antimalarials - are they effective and safe in rheumatic diseases? Reumatologia 56, 164-173 (2018).

[2]Rosenthal, A. R., Kolb, H., Bergsma, D., Huxsoll, D. \& Hopkins, J. L. Chloroquine retinopathy in the rhesus monkey. Invest. Ophthalmol. Vis. Sci. 17, 1158-1175 (1978).

[3] Modi, Y. S. \& Singh, R. P. Bull's-Eye Maculopathy Associated with Hydroxychloroquine. N. Engl. J. Med. 380, 1656 (2019).

[4] Sparrow, J. R., Duncker, T., Schuerch, K., Paavo, M. \& de Carvalho, J. R. L. J. Lessons learned from quantitative fundus autofluorescence. Prog. Retin. Eye Res. 74, 100774 (2020).

[5] Greenstein, V. C. et al. Quantitative Fundus Autofluorescence in HCQ Retinopathy. Invest. Ophthalmol. Vis. Sci. 61, 41 (2020).

[6] Reichel, C. et al. Quantitative Fundus Autofluorescence in Systemic Chloroquine/Hydroxychloroquine Therapy. Transl. Vis. Sci. Technol. 9, 42 (2020).

[7] Yusuf, I. H., Sharma, S., Luqmani, R. \& Downes, S. M. Hydroxychloroquine retinopathy. Eye (Lond). 31, 828-845 (2017).

Disclosure of Interests: Salvatore Parrulli: None declared, Mariano Cozzi Grant/research support from: Bayer, Nidek, Zeiss, Matteo Airaldi: None declared, Francesco Romano: None declared, Francesco Viola: None declared, Piercarlo Sarzi-Puttini: None declared, Giovanni Staurenghi Grant/research support from Heidelberg Engineering (C), QuantelMedical (C), Centervue (C), Carl Zeiss Meditec (C), Alcon (C), Allergan (C), Bayer (C), Boheringer (C), Genentech (C), GSK (C),Novartis (C), and Roche (C), Optos (F), Optovue (F) and Centervue (F), Alessandro Invernizzi Grant/research support from: Novartis (C), Bayer (C) DOI: 10.1136/annrheumdis-2021-eular.2022

\section{POS1394 ACCURACY AND PERFORMANCE OF A HANDHELD ULTRASOUND DEVICE TO ASSESS ARTICULAR AND PERIARTICULAR PATHOLOGIES IN PATIENTS WITH INFLAMMATORY ARTHRITIS}

G. Corte $^{1,2}$, S. Bayat ${ }^{1,2}$, K. Tascilar ${ }^{1,2}$, L. Valor ${ }^{1,2}$, L. Schuster ${ }^{1,2}$, J. Knitza $^{1,2}$, G. Schett ${ }^{1,2}$, A. Kleyer ${ }^{1,2}$, D. Simon ${ }^{1,2}$ on behalf of https://www.medizin3. uk-erlangen.de/en/research/research-main-focus/digital-rheumatologyand-experimental-imaging/. ${ }^{1}$ Friedrich-Alexander-Universität (FAU) and Universitätsklinikum Erlangen, Internal Medicine 3, Rheumatology and Immunology, Erlangen, Germany; ${ }^{2}$ Friedrich-Alexander University (FAU) Erlangen-Nürnberg and Universitätsklinikum Erlangen, Deutsches Zentrum Immuntherapie, Erlangen, Germany

Background: Handheld ultrasound (HHUS) devices have increasingly found their way into clinical practice due to several advantages (e.g. portability, sig nificantly lower purchase cost). However, there is no evidence to date on the accuracy and performance of HHUS in patients with inflammatory arthritis (IA). Objectives: To assess accuracy and performance of a new HHUS machine in comparison to a conventional cart-based sonographic machine in patients with IA Methods: Consecutive IA patients of our outpatient clinic with at least one tender and swollen joint in the 66/68 joint count were enrolled. US was performed on clinically affected joints with corresponding tendons/entheses using a cart-based sonographic device ("Samsung HS40") and a HHUS device ("Butterfly iQ") in standard scan positions. One blinded reader scored all images for the presence of following pathologic findings: erosions, bony enlargement, synovial hyperthrophy, joint effusion, bursitis, tenosynovitis and enthesitis. In addition, synovitis was graded (B Mode and power Doppler (PD)) by the 4-level EULAR-OMERACT scale [1]. To avoid bias by the blinded reader, who otherwise would have been tempted to identify pathological findings for each examined joint, we also included 67 joints of two healthy volunteers into the evaluation. We calculated the overall concordance and the concordance by type of joint and type of pathological finding between the two devices (percentage of observation pairs in which the same rating was given by both devices). The Cohen's kappa coefficient ( $\kappa$ ) with 95\% bootstrap confidence intervals was used to assess the agreement between the two US devices. We also measured the time required for the US examination of one joint with both devices.

Results: 32 patients (20 rheumatoid arthritis, 10 psoriatic arthritis, 1 gouty arthritis, 1 systemic lupus erythematosus) were included in this study. Mean age of patients was $58.2 \pm 13.7$ years, $63 \%$ were females. In total 186 joints were examined. The overall raw concordance in B-mode between the two devices was $97 \%$, with an overal кappa for agreement of $0.90,95 \% \mathrm{Cl}(0.89,0.94)$. No significant differences were found in relation to type of joint or pathological finding examined. The PD-mode of the HHUS device did not detect any PD-signal, whereas the cart-based device detected a PD-signal in 61 joints (33\%). The portable device did not offer any time saving compared to the cart-based device (mean time in seconds per examined region: 47 seconds for the HHUS device versus 46.3 seconds for the cart-based device) Conclusion: The HHUS device "Butterfly iQ" has been shown to be accurate in the assessment of structural joint damage and inflammation in patients with IA, 